\title{
REAÇÃO DE BAYLIS-HILLMAN: UMA ESTRATÉGIA PARA A PREPARAÇÃO DE INTERMEDIÁRIOS MULTIFUNCIONALIZADOS PARA SÍNTESE ORGÂNICA
}

\section{Fernando Coelho}

Instituto de Química - Depto. de Química Orgânica - UNICAMP - Cidade Universitária Zeferino Vaz - 13083 - 970 - Campinas - SP Wanda P. Almeida

Instituto de Ciências da Saúde - Depto. de Farmácia e Bioquímica - Universidade Paulista - Campinas - SP

Recebido em 17/11/98; aceito em 23/3/99

\begin{abstract}
THE BAYLIS-HILLMAN REACTION: A STRATEGY FOR THE PREPARATION OF MULTIFUNCTIONALISED INTERMEDIATES FOR ORGANIC SYNTHESIS. The Baylis-Hillman reaction has significantly advanced in the last ten years as demonstrated by a number of applications described in the literature. In this report we show some aspects of this reaction, including scope, limitations and perspectives.
\end{abstract}

Keywords: Baylis-Hillman; Morita-Baylis-Hillman; acrylate.

\section{INTRODUÇÃO}

\section{Definição}

A reação de Baylis-Hillman pode, em alguns aspectos, ser equiparável a algumas metodologias para a obtenção de substâncias $\beta$-hidroxicarboniladas. Numerosas publicações têm aparecido recentemente na literatura, mas ela é pouco conhecida da comunidade, e com este artigo esperamos torná-la mais popular, auxiliando na compreensão de numerosas publicações recentes. A reação, conhecida desde $1972^{1}$, pode ser definida como uma reação que resulta na formação de uma ligação carbono-carbono entre carbonos eletrofílicos $\mathrm{sp}^{2}$ (geralmente um aldeído) e a posição $\alpha$ de uma olefina contendo um grupo retirador de elétrons, EWG, (Esquema 1), ativada por um catalisador.

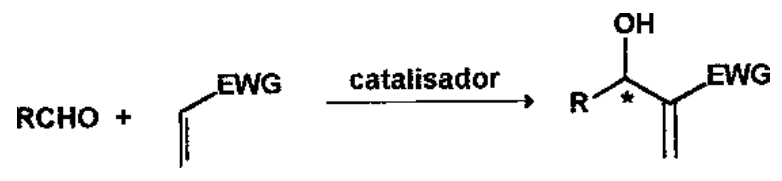

Esquema 1. A Reação de Baylis-Hillman.

O catalisador mais empregado é o 1,4-diazabiciclo [2.2.2.] octano (DABCO).

Esta reação, nas versões inter e intramolecular, vem recebendo considerável atenção por parte dos químicos orgânicos ${ }^{2}$, basicamente por apresentar características fundamentais para a eficiência de um método sintético: é regio, quimio e estereosseletiva; é econômica, requer condições brandas e providencia moléculas polifuncionalizadas que, através de sucessivas interconversões de grupos funcionais, podem permitir o acesso a importantes intermediários sintéticos. A potencialidade desta reação é muito grande, particularmente no que se refere ao controle estereoquímico. Deve-se observar que a formação do aduto leva à criação de pelo menos um centro estereogênico. Embora esta transformação seja mais conhecida como reação de Baylis-Hillman, deve-se grande parte do crédito a Morita ${ }^{2 b}$, que investigou o uso de fosfinas como catalisadores, ao invés de DABCO. Assim, ela é também conhecida como reação de Morita-Baylis-Hillman.

coelho@iqm.unicamp.br

almeida@iqm.unicamp.br

\section{Mecanismo de Reação ${ }^{3,4}$}

O Esquema 2 representa o mecanismo mais aceito. Em uma primeira etapa, considerada a etapa lenta ${ }^{4 b}$, ocorre uma adição de Michael do catalisador (amina terciária I ou fosfina) ao sistema $\alpha, \beta$-insaturado II, gerando o zwitterion III. A condensação aldólica entre III e o aldeído IV leva ao alcóxido $\mathbf{V}$, que sofre uma transferência de próton, fornecendo o enolato VI. Neste estágio, a decomposição deste intermediário dá o produto $\beta$-hidroxi- $\alpha$-metileno carbonilado VII, regenerando o catalisador I. Este Esquema mecanístico é apenas uma simplificação e os estudos têm demonstrado que a natureza do aldeído e do nucleófilo irá determinar a reversibilidade ou não das etapas do ciclo catalítico.
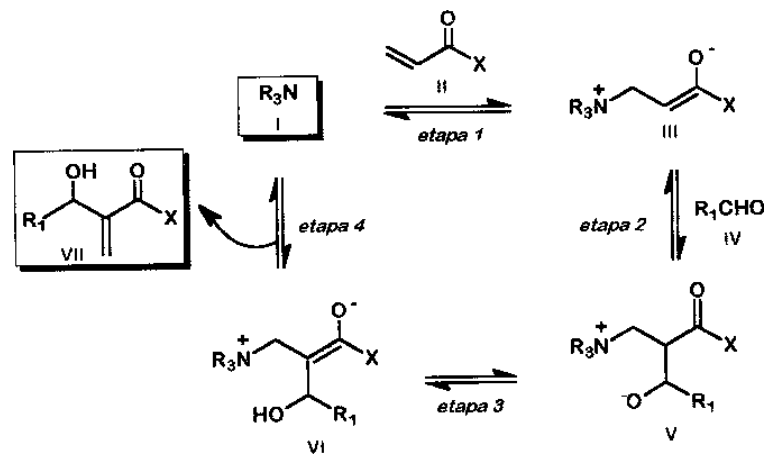

Esquema 2. Etapas Envolvidas no Mecanismo da Reação de Baylis-Hillman.

\section{REATIVIDADE E LIMITAÇÕES}

Nesta reação os eletrófilos podem ser aldiminas ${ }^{5}$ ou aldeídos, aromáticos ou alifáticos. Cetonas e cetoésteres também são usados, mas exigem condições especiais, como por exemplo, pressurização. Os aldeídos alifáticos reagem mais rapidamente que os aromáticos, e estes são melhores que as aldiminas, cetonas e cetoésteres. Em relação aos nucleófilos, os acrilatos (EWG $=\mathrm{CO}_{2} \mathrm{R}$ ) são empregados com mais frequência. Os acrilatos arílicos são os melhores nucleófilos, segundo Caubère e cols. ${ }^{4 a}$, mas podem causar irritação, ânsia de vômito e dores de cabeça em pessoas mais sensíveis. Enonas também podem ser utilizadas como nucleófilos em reações de 
Baylis-Hillman, e a reação é bem sucedida, podendo ser catalisada por aminas terciárias, fosfinas, e outros catalisadores de ródio ou rutênio ${ }^{6-9}$. As adições destas cetonas catalisadas por aminas são muito "limpas" quando realizadas em solvente, geralmente tetraidrofurano. Enonas contendo substituintes na posição $\alpha$ reagem mais lentamente do que as não ramificadas. Quanto às enonas cíclicas existe apenas um relato na literatu$\mathrm{ra}^{10}$, no qual a cicloexenona foi submetida à reação com 4nitrobenzaldeído, mas apenas em condições especiais, na presença de ácido de Lewis e catalisadores de enxofre ou selênio.

Nitrilas, aldeídos, sulfonas, sulfonatos e fosfonatos $\alpha, \beta$ insaturados também são utilizados.

O tempo desta reação pode variar de alguns minutos até 30 dias. Assim, procura-se manipular fatores externos visando otimizar o tempo de reação. Pressurização ${ }^{11}$ e sonicação $^{12}$ são as técnicas mais empregadas para a diminuição do tempo de reação. Pressões superiores à atmosférica têm permitido não só o aumento da velocidade de reação, mas também o aumento da seletividade (discussão posterior) e rendimento químico. O uso de microondas ${ }^{13}$, também é cada vez mais rotineiro nas reações de Baylis-Hillman, reduzindo consideravelmente o tempo de reação. A natureza do solvente, quando há necessidade de usá-lo, também afeta a velocidade, assim como a adição de sais para os solventes aquosos. Já a temperatura de reação pouco tem influenciado nos resultados.

\section{VERSÃO ASSIMÉTRICA}

\section{Olefinas Quirais (nucleófilos)}

- acrilatos quirais: o acesso fácil a vários tipos de acrilatos quirais fez desta abordagem a principal no estudo da versão assimétrica da reação de Baylis-Hillman. Basavaiah e cols. $^{2 a}$ investigaram uma série de acrilatos quirais derivados do mentol, prolinol e outros. Os excessos diastereoisoméricos variaram de 7 a $70 \%$. Entretanto, o excesso diastereoisomérico é dependente da pressão ${ }^{14}$ : acrilato de Lmentila e benzaldeído, por exemplo, reagem a uma pressão de 7,5 Kbar, fornecendo um único diastereoisômero. À pressão atmosférica, o excesso diastereoisomérico foi de $22 \%$.

- auxiliar do Oppolzer: mais recentemente, Leahy e cols. ${ }^{15}$ relataram o uso do nucleófilo VIII derivado deste auxiliar (Figura 1), em reações de Baylis-Hillman com vários aldeídos. Após remoção do auxiliar nos adutos, estes apresentaram excessos enantioméricos maiores que 99\%. Entretanto, a reação de VIII com benzaldeído não funcionou, constituindo-se, até o momento, a limitação da metodologia.

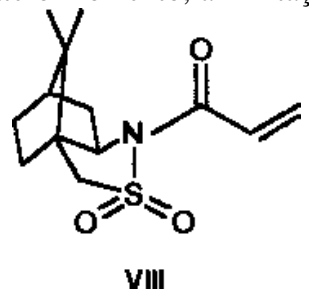

Figura 1. Nucleófilo quiral utilizado em reações de Baylis-Hillman.

\section{Eletrófilos Quirais (aldeídos)}

Dos vários eletrófilos em reações de Baylis-Hillman, apenas aldeídos quirais foram estudados. Dentre estes destacam-se os $\alpha$ e $\beta$ - alcoxialdeídos ${ }^{16}$, e alguns $\alpha$-aminoaldeídos ${ }^{17}$. Em ambos os casos, foi constatada uma preferência pelo isômero anti, explicada pelo modelo de Felkin-Ahn ${ }^{18}$, embora a diastereosseletividade não tenha sido muito alta ( 70:30).

Kundig e cols. ${ }^{19}$ empregaram benzaldeídos e ariliminas complexados com tricarbonilcromio como eletrófilos em reações de Baylis-Hillman. Estas procederam com diastereosseletividades surpreendemente altas (95\% ed).

\section{Catalisadores Quirais}

Atualmente, o desenvolvimento de reações catalíticas assimétricas é um desafio para o químico orgânico. Na reação de Baylis-Hillman, os principais catalisadores empregados são aminas terciárias, que participam em todas as etapas da reação, incluindo-se aquela em que o centro estereogênico é criado. Consequentemente, se a amina é quiral ela pode promover uma indução assimétrica. Com base nestes conceitos, uma variedade de aminas terciárias oticamente ativas foi estudada, como por exemplo a quinina, quinidina, cinchonidina e retronecina ${ }^{14,20}$, mas os excessos enantioméricos mantiveram-se entre 10 e $40 \%$. A amina IX (Figura 2) foi a que promoveu o maior excesso enantiomérico $(47 \%)$, mas somente na reação entre o 4-nitrobenzaldeído e a metilvinilcetona, empregando-se $5 \mathrm{Kbar}$ de pressão ${ }^{21}$.

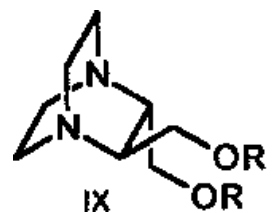

\section{$\mathbf{R}=$ benzila, arila, TBDMS, TBPS}

Figura 2. Catalisador quiral usado em reações de Baylis-Hillman.

\section{CARACTERÍSTICAS EXPERIMENTAIS}

A reação de Baylis-Hillman é experimentalmente muito mais simples do que reações análogas. As características às quais nos referimos são:

- na maioria dos casos, não há necessidade de solventes. Quando são necessários, os mais usados são: metanol, diclorometano, tetraidrofurano e dioxana, sem tratamento prévio, ou secagem. A água também é utilizada quando o aldeído é solúvel.

- temperatura: a maioria das reações ocorre à temperatura ambiente, sem que este fator afete a seletividade da reação, no caso da versão assimétrica.

- atmosfera: também não há necessidade de atmosfera inerte, sendo recomendada apenas a troca de atmosfera quando o aldeído é muito sensível ao oxigênio atmosférico.

- no caso de necessidade de ultrassom, equipamentos simples do tipo Cleaner, utilizados na limpeza de instrumentos, funcionam bem, e para irradiação microondas, um equipamento doméstico é suficiente.

- escala: pode variar de $1 \mathrm{mmol}$ até $0,5 \mathrm{~mol}$

- catalisador: utilizado na proporção de 10 a 30\%. Quantidades equimolares não alteram os resultados, segundo a literatura ${ }^{2}$

- rendimentos químicos: embora não sejam muito altos $(\sim 70 \%)$, na maioria dos casos o aldeído é recuperado quase quantitativamente, podendo ser reciclado. Os adutos costumam ser estáveis frente às técnicas rotineiras de purificação, como por exemplo, coluna cromatográfica à pressão normal ou reduzida.

\section{APLICAÇÕES EM SÍNTESE}

Os adutos de Baylis-Hillman apresentam no mínimo três grupos funcionais: um grupo hidroxila, uma olefina e um éster, cetona, nitrila, sulfona ou fosfonato, além de um centro estereogênico. Explorando a reatividade destes grupos funcionais, podemos ter acesso a importantes intermediários.

\section{Síntese Estereosseletiva de Olefinas}

O grupo $\alpha$-metileno presente no aduto de Baylis-Hillman tem permitido a utilização destes intermediários na síntese de olefinas trissubstituídas, destacando-se o trabalho de Hoffmann e Buchholz ${ }^{22}$. Por exemplo, o aduto VII (Esquema 3) forneceu 
o brometo alílico VIII, após o tratamento com $\mathrm{HBr} / \mathrm{H}_{2} \mathrm{SO}_{4}$ concentrado. A reação foi regio e $Z$-estereosseletiva. Entretanto, a substituição do grupo carbometoxi por um grupo ciano, leva à perda da seletividade Z. Os rendimentos em ambos os casos variaram de 70 a $91 \%$. Estes intermediários foram utilizados na síntese de aminoácidos não proteinogênicos ${ }^{22}$.

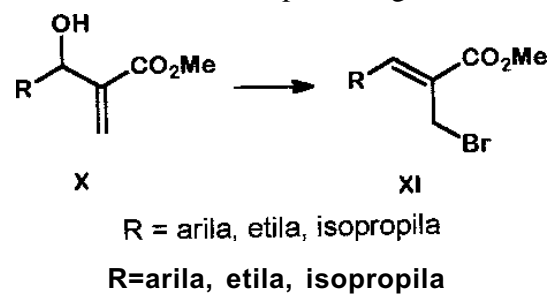

Esquema 3. Obtenção de Olefinas Trissubstituídas.

\section{Adições Nucleofílicas à Ligação Dupla}

As reações de adição à ligação dupla $(1,4)$ são geralmente acompanhadas por uma reação de eliminação, caracterizando um processo do tipo $S_{N} 2^{\prime}$, mas algumas adições 1,4 estão descritas na literatura, conforme comentários seguintes.

Vários nucleófilos tem sido utilizados em reações de adição ao grupo metileno. Dentre estes destacam-se os nucleófilos de carbono e aminas.

No primeiro grupo de nucleófilos, observou-se que cupratos mistos $^{23}$ e reagentes de Grignard na presença de sais de cobre ${ }^{24}$, levam aos produtos resultantes de reações do tipo $S_{N} 2$ '. Enolatos derivados de substâncias 1,3-dicarboniladas podem ou não levar a este tipo de produto ${ }^{25}$ A configuração da ligação dupla formada irá depender da natureza do grupo retirador de elétrons, tal qual no caso anterior.

Em trabalho recente, ${ }^{12 b}$ pudemos verificar que a adição de cianeto ao aduto XII (Esquema 4) derivado do piperonal e acrilato de metila, ocorre em bons rendimentos, sem rearranjos. Constatamos também que a configuração relativa dos centros estereogênicos formados era uma consequência da hidroxila estar ou não protegida: hidroxila livre (XIIIa) leva predominantemente ao aduto anti, enquanto a sua proteção na forma de éter de silício (TBDMS, XIIIb) favorece o isômero syn.

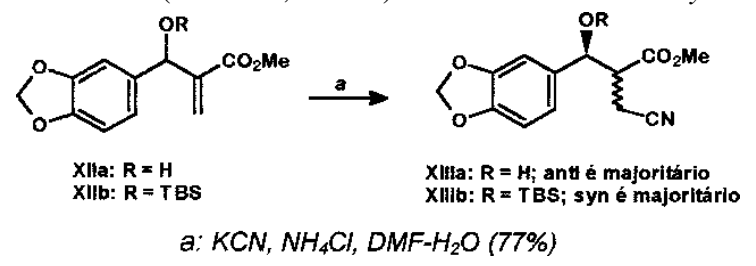

a: $\mathrm{KCN}, \mathrm{NH}_{4} \mathrm{Cl}, \mathrm{DMF}-\mathrm{H}_{2} \mathrm{O}(77 \%)$

Esquema 4. Adição de Cianeto a Aduto de Baylis-Hillman.

A seletividade syn, também foi observada por Perlmutter e Tabone $^{26}$ na adição de aminas a adutos de Baylis-Hillman.

\section{Outras Reações}

Adutos de Baylis-Hillman têm sido utilizados também em reações de redução (hidrogenação catalítica homogênea, principalmente $)^{27}$, diidroxilação ${ }^{27 b}$ e reações de Diels-Alder ${ }^{28}$, além da adição de radicais livres ${ }^{29}$.

\section{PERSPECTIVAS FUTURAS}

Nos 26 anos decorridos desde o seu patenteamento, a reação de Baylis-Hillman teve sem dúvida um desenvolvimento notório.

As suas características experimentais refletem aspectos que não podem ser ignorados, quando da elaboração de uma proposta sintética, tais como o custo e disponibilidade dos reagentes. No caso específico desta reação, a preparação de intermediários pode ser bem menos dispendiosa. Quando falamos em custo, devemos considerar não só a aquisição dos reagentes, mas também os solventes, hidretos e outros agentes utilizados em sua secagem, o que não é necessário na referida reação.

Entretanto, para que a reação de Baylis-Hillman possa entrar para o circuito das metodologias "usuais", aspectos fundamentais precisam ser investigados com maiores detalhes, tornando-a um campo fértil para a pesquisa. Vários parâmetros relacionados à velocidade de reação têm sido estudados. Recentemente, Aggarwal et.al. ${ }^{30}$, relataram a aceleração da reação na presença de ácidos de Lewis baseados em lantânio, escândio e samário, na forma de triflatos. Outros autores ${ }^{10}$ também descrevem o uso de ácidos de Lewis, principalmente tetracloreto de titânio, mas na presença de sulfetos e selenetos. A introdução de ácidos de Lewis na reação de Baylis-Hillman parece ser bastante promissora, observando-se os cuidados relativos à estequiometria da reação.

A área mais promissora é sem dúvida a da seletividade. Um avanço inegável foi a utilização do nucleófilo quiral, derivado do auxiliar do Oppolzer, mas esta estratégia ainda apresenta limitações, já que até o momento não se aplica a aldeídos aromáticos, além de ter um custo razoavelmente alto. Assim, a busca de novos auxiliares precisa ser continuada.

No campo da seletividade, a nossa pesquisa de novos auxiliares tem apresentado um ponto crítico, que é exatamente a obtenção do aduto em rendimentos químicos satisfatórios. Por exemplo: a acriloiloxazolidinona XIV (Esquema 5) e o acrilato $\mathbf{X V}$, não têm se apresentado como bons nucleófilos na reação de Baylis-Hillman, mas condições especiais continuam sendo investigadas.
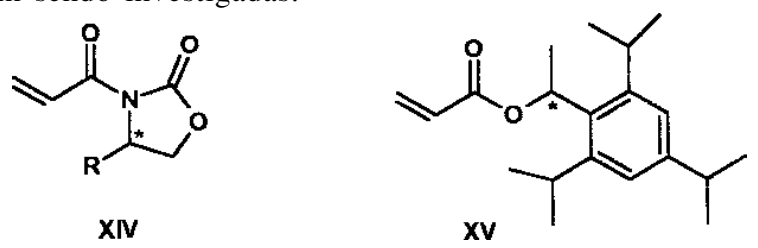

Esquema 5. Nucleófilos Investigados em Reação de Baylis-Hillman Assimétrica.

Além da pesquisa de novos auxiliares quirais para esta reação, uma outra estratégia no campo da seletividade é tão ou mais promissora: a busca de novos catalisadores, que possam levar à obtenção de adutos com alto grau de pureza enantiomética.

\section{REFERÊNCIAS}

1. Baylis, A. B.; Hillman, M. E. D.; Patente Alemã 2155113, 1972 (Chem. Abst. 1972, 77, 34174q).

2. a. Basavaiah, D.; Rao, P. D.; Hyma, R. S.; Tetrahedron 1996, 52, 8001; b. Ciganek, E.; In Organic Reactions 1997, 51, Cap. 2, 201-350.

3. Drewes, S. E.; Roos, G. H. P.; Tetrahedron 1988, 44, 4653.

4. a. Fort, Y.; Berthe, M. C.; Caubere, P.; Tetrahedron 1992, 48, 6371; b. Hill, J. S.; Isaacs, N. S. J.; Phys. Org. Chem. 1990, 3, 285; c. Hoffmann, H. M. R.; Rabe, J.; Angew. Chem. Int. Ed. Engl. 1983, 22, 795.

5. Takagi, M.; Yamamoto, K.; Tetrahedron 1991, 47, 8869 e referências citadas.

6. Amri, H.; Villieras, J.; Tetrahedron Lett. 1986, 27, 4307.

7. Drewes, S. E.; Freese, S. D.; Emslie, N. D.; Roos, G. H. P.; Synth. Commun. 1988, 18, 1565.

8. Sato, S.; Matsuda, I.; Shibata, M.; J. Organomet. Chem. 1989, 377, 347.

9. Basavaiah, D.; Bharathi, T. K.; Gowriswari, V. V. L.; Synth. Commun. 1987, 17, 1893. 
10. Kataoka, T.; Iwama, T.; Shin-ichiro, T.; Tatsunori, I.; Watanabe, Shin-ichi.; Tetrahedron 1998, 54, 11813.

11. a. Hill, J. S.; Isaacs, N. S.; Tetrahedron Lett. 1986, 27 , 5007; J. Chem. Res. (S), 1988, 330; b. Isaacs, N. S.; Tetrahedron 1991, 47, 8463.

12. a. Roos, G. H. P.; Rampersadh, P.; Synth. Commun. 1993, 23(9), 1261; b. Almeida, W. P.; Coelho, F.; Tetrahedron Lett. 1998, 8609.

13. Bhat, S. V.; Padmakumar, R.; Balu, N.; Mukherjee, S. B.; Kundu, M. K.; Synlett 1994, 444.

14. Gilbert, A.; Heritage, T. W.; Isaacs, N. S.; Tetrahedron: Asymmetry 1991, 2, 969.

15. a. Leahy, J. W.; Rafel, S.; Brzezinski, L. J.; J. Am. Chem. Soc. 1997, 119, 4317; b. Piber, M.; Leahy, J. W.; Tetrahedron Lett. 1998, 34, 2043.

16. Drewes, S. E.; Njamela, O. L.; Roos, G. H. P.; Chem. Ber. 1990, 123, 2455, e referências citadas.

17. a. Roos, G.; Manickum, T.; Synth. Commun. 1991, 21, 2269; b. Drewes, S. E.; Khan, A. A.; Rowland, K.; Synth. Commun. 1993, 23, 183.

18. a. Cherest, M.; Felkin, H.; Prudent, N.; Tetrahedron Lett. 1968, 18, 2199; b. Ahn, N. T.; Top. Curr. Chem. 1980, 88, 144.

19. Kundig, E. P.; Xu, L. H.; Schnell, B.; Synlett 1994, 413, e referências citadas

20. Drewes, S. E.; Roos, G. H. P.; Tetrahedron 1988, 44, 4653.

21. a. Oishi, T.; Hirama, M.; Tetrahedron Lett. 1992, 33, 639; b. Oishi, T.; Oguri, H.; Hirama, M.; Tetrahedron
Asymmetry 1995, 6, 1241; c. Markó, I. E.; Giles, P. R.; Hindley, N. J.; Tetrahedron 1997, 53, 1015.

22. Hoffmann, H. M. R.; Buchholz, R.; Helv. Chim. Acta 1991, 74, 1213.

23. Amri, H.; Rambaud, M.; Villieras, J.; J. Organomet. Chem. 1990, 384, 1.

24. Amri, H.; Rambaud, M.; Villieras, J.; Tetrahedron 1990, $46,3535$.

25. a. Beltaief, I.; Amri, H.; Synth. Commun. 1994, 24, 2003; b. Perlmutter, P.; Lawrence, R. M.; Chem. Lett. 1992, 305.

26. Perlmutter, O.; Tabone, M.; Tetrahedron Lett. 1988, $29,949$.

27. a. Leahy, J. W.; Rafel, S.; J. Org. Chem. 1997, 62, 1521; b. Brown, J. M.; Cutting, I.; James, A. P.; Bull. Soc. Chim. Fr. 1988, 211; c. Brown, J. M.; James, A. P.; Prior, L. M.; Tetrahedron Lett. 1987, 28, 2179; d. Brown, J. M.; Angew. Chem. Int. Ed. Engl. 1987, 26, 190; e. Kitamura, M.; Kasahara, I.; Manabe, K.; Noyori, R.; Takaya, H.; J. Org. Chem. 1988, 53, 708.

28. a. Hoffmann, H. M. R.; Eggert, U.; Poly, W.; Angew. Chem. Int. Ed. Engl. 1987, 26, 1015; b. Hoffmann, H. M. R.; Weichert, A.; Slawin, A. M. Z.; Tetrahedron 1990 46, 5591.

29. Mase, N.; Wake, Y.; Watanabe, Y.; Toru, T.; Tetrahedron Lett. 1998, 39, 5553.

30. Aggarwal, V. K.; Mereu, A.; Tarver, G. T.; McCague, R.; J. Org. Chem. 1998, 63, 7183. 